\title{
Research on the Adjustment and Control of Exercise Load in Sport Teaching
}

\author{
CHEN JINRONG ${ }^{1, a}$, LIU JING ${ }^{1, b}$ \\ ${ }^{1}$ Yunnan Yuxi normal university Sports college, 653100, China \\ a ceg@yxnu.net, ${ }^{b}$ liujing@yxnu.net
}

Keywords: Physical education teaching; Exercise load; Optimal distribution combination; Information feedback control; Exercise physiology; Parameter optimization

\begin{abstract}
The determination of exercise load in sports teaching process has great significance. if the exercise load arrangement is too small, then we can not reach the purpose of keeping fit. If the exercise load is too large, as a result, it will cause great harm to the body. This paper combined with the exercise physiology and the optimal distribution control theory, at the same time took the mission and goals of the Physical Education teaching into consideration. On these bases this paper made a thorough study of the exercise load's adjustment and control of sports teaching.
\end{abstract}

\section{Introduction}

The arrangement of exercise load in sports teaching is mainly related to the arrangement of exercise time and the movement task. At present the arrangement of exercise load in sports teaching is generally determined by teachers who made it through experience and sports [1-3]. The determination of load and movement effect has not experienced specifically quantitative analysis and qualitative research. So it has no scientific basis, and can not guarantee the quality of student movement in physical education as well as to achieve the purpose of keeping fit. On the contrary, it reduced the physical education teaching level and quality. In this background, this paper made a deep research for the exercise load arrangement and control in sports teaching process [4]. In recent years, virtual reality technology application in sports has intellectualized simulation, qualitative and quantitative simulation, distributed interactive simulation, visual simulation, multi-media simulation and so on. The simulation is based on virtual reality technology, which focuses on a variety of perception, interaction and immersion. As the physical training requires athletes' variety of feeling (auditory, tactile, olfactory, visual) participatory role, simulation based on virtual reality technology will surely be in-depth mining application in sports field. And it made a quantitative analysis for the determination of the exercise load mainly through the arrangement of the capacity from the heart of the course of the campaign, as well as the school physical education curriculum task.

This paper received the average curve of the students' physical education curriculum changes in heart rate in the way of survey, and it combined with the optimal allocation of portfolio theory made a parameter optimization of the physical education curriculum load. So ultimately it got the best sports teaching exercise load distribution ratio.

\section{Optimal exercise physiology principle}

The information era and network technology not only speed up the process of economic globalization, but also continually promote the rapid development and application of information 
technology. At present, education and information technology are closely related, and is combined with the level of information technology application will contribute to improved education that is also as everyone knows. Therefore, how to achieve information technology in sports training to enhance the role, it will be further exploration, getting the conclusion will help guidance in the physical training with some reference value and practical significance.

In order to study the physiology reaction of exercise load, these papers investigate and count 3,000 students in 60 schools. Through the arrangement for sports in physical education we can collective train the students, and use the cardiopulmonary function tester to entirely monitor the movement process during the collective training process [5]. The average level of students' heart rate change in class is not steady. The students' heart rate is small in the preparation before class, while with the students moving load increases in the class their heart rate gradually increased. And at the end of the class the amount of students' heart rate gradually decreased with the reduction of their moving load. Changes in the average heart rate of the students in the class concentrated between 120 and 135. Therefore, in the sports teaching process, the load arrangements in class should in this grade range as much as possible. The load intensity is relatively high in the sports of football and basketball programs, their heart rate close to 130. Therefore the arrangement of the Project's load time should be as reasonable as possible to avoid the student body being injury.

\section{Optimal allocation of exercise load control mathematical model}

The adjustment and control of the exercise load in sports teaching used load incremental form. As the load size was arranged randomly, so the effect of the exercise load changes randomly. The exercise load's effect and size is one-to-one relationship. Virtual reality technology has been widely used in competitive sports as a high-tech. According to the simulation of 3D motion information, it can draw up training plan, and to have a scientific assessment and reasonable choice by collecting various information, such as collecting the psychological and physiological index of athletes, training environment and simulating opponent. Therefore, a variety of scientific research project based on virtual reality technology has been paid more attention because of its remarkable effects. Virtual reality technology can be served in sports training. It is of great significance towards the development of competitive sports. The initial establishment of kinematics models, directly reflecting the real time state of athletes, inspiring athletes to challenge self-limits, promote the development of China's sports system. On the assumption that the arrangement of the physical education curriculum have " $x$ " kinds of effects, we separate the initial course of the entire sports exercise load "y " into " $\mathrm{x}$ " parts, and each exercise load corresponds to a physical effect, assume that $Y_{k 0}$ is the Loading dose of the movement of $k$.

$$
Y_{0}=\sum_{l=1}^{n} Y_{l 0}
$$

On the assumption that ${ }^{v_{l}}$ is the load ratio in the movement of $k$, and

$$
\sum_{l=1}^{n} v_{l}=1
$$


On the assumption that ${ }^{u_{l}}$ represents the physical effect of the movement of $k$, so the motion effects generated by $k$ can be expressed as:

$$
Y_{l} v_{l}\left(1+u_{l}\right)
$$

The overall effect of physical load:

$$
\sum_{l=1}^{n} Y_{l} v_{l}\left(1+u_{l}\right)
$$

The exercise load effect variance of the two combinations of sports programs $E$ and $F$ can be expressed as:

$$
\operatorname{Var}\left(u_{q}\right)=v_{E}^{2} \sigma_{E}^{2}+2 v_{E} v_{E} \sigma_{E F}+v_{E}^{2} \sigma_{E}^{2}
$$

Among

$$
\sigma=\sum_{m=1}^{n} \sum_{k=1}^{n} v_{k} v_{m} \sigma_{k m}
$$

In which ${ }^{\sigma_{k m}}$ represents covariance. The overall effect of the exercise load can be represented by matrix, and the covariance matrix can be expressed as:

$$
Z=\left(\begin{array}{cccc}
\operatorname{Var}\left(\bar{u}_{1}\right) & \operatorname{Cov}\left(\bar{u}_{1}, \bar{u}_{2}\right) & \cdots & \operatorname{Cov}\left(\bar{u}_{1}, \bar{u}_{N}\right) \\
\operatorname{Cov}\left(\bar{u}_{2}, \bar{u}_{1}\right) & \operatorname{Var}\left(\bar{u}_{2}\right) & \cdots & \operatorname{Cov}\left(\bar{u}_{2}, \bar{u}_{N}\right) \\
\vdots & \vdots & \ddots & \vdots \\
\operatorname{Cov}\left(\bar{u}_{N}, \bar{u}_{1}\right) & \operatorname{Cov}\left(\bar{u}_{N}, \bar{u}_{2}\right) & \cdots & \operatorname{Var}\left(\bar{u}_{2}\right)
\end{array}\right)
$$

The determination of exercise load in sports teaching based primarily on the capacity of the heart for the sport, which is associated with physical fitness and sports factors. Physiology and kinematics of theoretical knowledge can be applied to give the exercise load of the initial motion of the hearth a judgment.

The heart rate affordability of boys and girls is different. Therefore the arrangement of exercise load should be treated according to the gender-specific. The highest level in the table is beyond 180, therefore when we making the teaching goal and the teaching tasks we should avoid the strenuous exercise level reached 1 or 2 level. A exercise load body feeling table which was designed by Borg--a Swiss physiologis divided the body feeling when people doing exercise into 5-20 grades.

According to the capacity of exercise load heart rate and exercise load body feeling table and through the second part of the mathematical model, the paper received the parameters of $\sigma=\sum_{\mathrm{m}=1}^{n} \sum_{k=1}^{n} v_{k} v_{m} \sigma_{k m}$. ${ }^{i}$ compared the parameters and first try motion parameters according to the feedback information principle. After optimizing the parameters, $i$ finally can get the sports course time load schedule.

The exercise load level is relatively small in the pre-class preparation and before class over. Students' exercise load level is small before class which can help students better into the sport. It will hurt the students' body, if they start exercising too violent. The arrangements of reducing the amount of exercise at the end of the class can help students have a sufficient rest, so it makes the body relax. 
Schools progressively increase the amount of exercise load in the class which is Consistent with the second part of the mathematical model. And it proved the effectiveness of the optimal portfolio allocation method in the study of sports teaching load adjustment and control.

As long as we analysed seriously and think in different parts of the school physical education situation, study the different school sports development characteristic and law, strengthen the physical education teaching contents and process optimization, layer upon layer takes seriously, catch condominium together, can actually implement quality education, improve students' physical culture and accomplishment, enhance the body quality of students, thus facilitating culture teaching quality improved steadily, achieve the improvement of students' physical quality, promotes the student morality, intelligence, body, beauty, work full scale development, ultimately to achieve the purpose of educating people, rural school physical education will have a new situation, can truly nationwide to promote quality education, for the construction of a harmonious society to provide strong in support.

\section{Conclusion}

This paper makes a thorough inquiry for the exercise load's adjustment and control in sports teaching by combining with mathematical statistics method and control mathematical model. In order to study the students' physiological changes in physical education curriculum, we collective train the students in more than 60 schools. Through the statistics of 3000 student movement process physiological reaction we found that the changes of student's heart rate is unstable distributed. The statistics shows that the highest heart rate is up to 140, and the average heart rates of the schools are concentrated in the 120135. Besides different sports will show different heart rate, and the average heart rate in football process can reach 130. Finally, the paper made a parameter optimization by using the model of the optimal combination of load control and combined with the arrangement of Sports course load which was made by the capacity of exercise load heart rate and exercise load body feeling table. As a result, it can get the ultimate time scale of the exercise load in sports teaching class which provides a theoretical basis for the arrangement of sports load in sports courses.

\section{References}

[1] Su Rui, Xun Ma, "The Impact of Title Event Sponsorship Announcements on Enterprise incomings Based on Background of China", AISS, Vol. 4, No. 19, pp. 438-442, 2012.

[2] Ester Cerin, "Predictors of competitive anxiety direction in male Tae Kwon Do practitioners: a multilevel mixed idiographic/nomothetic interactional approach", Psychology of Sport and Exercise, Vol. 5, No. 4, pp. 497-516, 2005.

[3] Jonathan Weiss, Terry Diamond, Jenny Demark, "Involvement in Special Olympics and its relations to self-concept and actual competency in participants with developmental disabilities", Research in Developmental Disabilities, Vol. 24, No. 4, pp. 281-287, 2007.

[4] Fang Ya, "The Modeling on Sports Videos based on Bayesian network and Feature Maps", IJACT, Vol. 4, No. 22, pp. 692-608, 2012.

[5] Sean P. Cumming, Ronald E. Smith, Frank L. Smoll, "Development and validation of the Achievement Goal Scale for Youth Sports", Psychology of Sport and Exercise, Vol. 9, No. 5, pp. 686-692, 2008. 